\title{
Simultaneous reconstruction of the forearm extensor compartment tendon, soft tissue, and skin
}

\author{
Jeongseok $\mathrm{Oh}^{1}$, Hee Chang $\mathrm{Ahn}^{1}$, Kwang Hyun Lee ${ }^{2}$ \\ Departments of ${ }^{1}$ Plastic and Reconstructive Surgery and ${ }^{2}$ Orthopedic Surgery, Hanyang University College of Medicine, Seoul, Korea
}

\begin{abstract}
Malignant peripheral nerve sheath tumor (MPNST) is a very rare type of sarcoma, with an incidence of $0.001 \%$. MPNST has a 5 -year survival rate near $80 \%$, so successful reconstruction techniques are important to ensure the patient's quality of life. Sarcoma of the forearm is known for its poor prognosis, which leads to wider excision, making reconstruction even more challenging due to the unique anatomical structure and delicate function of the forearm. A 44-year-old male presented with a large mass that had two aspects, measuring $9 \times 6$ $\mathrm{cm}$ and $7 \times 5 \mathrm{~cm}$, on the dorsal aspect of the right forearm. The extensor compartment muscles (EDM, EDC, EIP, EPB, EPL, ECRB, ECRL, APL) and invaded radius were resected with the mass. Tendon transfer of the entire extensor compartment with skin defect coverage using a $24 \times 8 \mathrm{~cm}$ anterolateral thigh (ALT) perforator free flap was performed. The patient was discharged after 18 days without wound complications, and has not complained of discomfort during supination, pronation, or wrist extension/flexion through 3 years of follow-up. To our knowledge, this is the first report of successful reconstruction of the entire forearm extensor compartment with ALT free flap coverage after resection of MPNST.
\end{abstract}

Keywords Forearm / Neoplasms / Surgical flaps
Correspondence: Hee Chang Ahn Department of Plastic and Reconstructive Surgery, Hanyang University College of Medicine, 222-1 Wangsimni-ro, Seongdong-gu, Seoul 04763, Korea

Tel: $+82-2-2290-8560$

Fax: +82-2-2295-7671

E-mail: ahnhc@hanyang.ac.kr

Received: 18 Dec 2017 • Revised: 30 Apr 2018 • Accepted: 17 May 2018

pISSN: 2234-6163 • elSSN: 2234-6171 • https://doi.org/10.5999/aps.2017.01802 • Arch Plast Surg 2018;45:479-483

\section{INTRODUCTION}

Malignant peripheral nerve sheath tumor (MPNST) is a very rare type of sarcoma associated with neurofibromatosis type 1 (NF1) or exposure to radiation $[1,2]$. Multidisciplinary treatment with chemotherapy, radiotherapy, and limb-salvage surgery is required for MPNST. Forearm reconstruction after sarcoma resection requires soft tissue coverage and functional recovery. Anterolateral thigh (ALT) free flaps have gained popularity due to their versatility and convenience [3]. We report this case as a demonstration that simultaneous reconstruction of the forearm extensor compartment tendon, soft tissue, and skin is a viable option. The patient described in this study provided written informed consent for this publication.

\section{CASE}

A 44-year-old male patient, who had twice suffered from recurrent sarcoma of the right forearm, presented to Hanyang University Medical Center with a large mass that had two aspects, measuring $9 \times 6 \mathrm{~cm}$ and $7 \times 5 \mathrm{~cm}$, on the dorsal aspect of the right forearm (Fig. 1). The patient was otherwise healthy, had no family history of neurofibroma, and had not been exposed to radiation. Excision and primary closure had been performed previously, and medical records only reported the tumor to be a sarcoma, with no indication of the subtype. The patient only complained of mild tingling sensations and did not complain of any discomfort during supination, pronation, or wrist extension/flexion. Positron emission tomography-computed tomog- 
Fig. 1. Preoperative design of resection and tendon transfer An anterolateral thigh perforator flap was planned for coverage of the predicted skin defect.

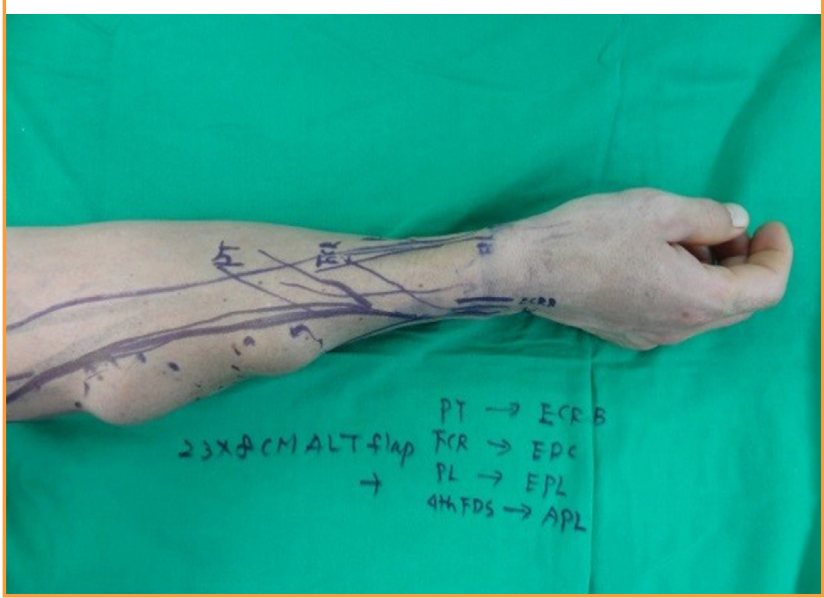

\section{Fig. 2. Immediate postoperatve photograph}

An anterolateral thigh perforator flap measuring $24 \times 8 \mathrm{~cm}$ was used for skin coverage.

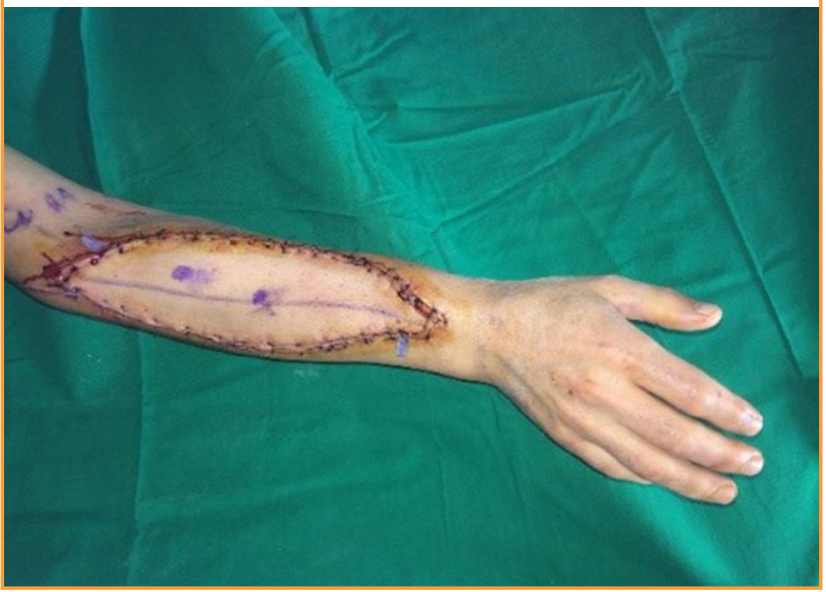

raphy revealed a hypermetabolic soft tissue mass in the right forearm muscle layer, suggesting malignancy. Magnetic resonance imaging showed a fusiform, well-circumscribed, well-enhanced non-homogenous soft tissue mass in the right forearm with invasion of the extensor carpi radialis muscle and extensor digitorum muscle (T2bN0M0). The extensor compartment muscles (EDM, EDC, EIP, EPB, EPL, ECRB, ECRL, APL) and invaded radial bone were resected along with the mass. A preoperatively planned tendon transfer was performed (Fig. 1). The pronator teres was transferred to the extensor carpi radialis brevis to prevent wrist drop. Transfers of the flexor carpi radialis to the extensor digitorum communis, third flexor digitorum superficialis to the extensor pollicis longus, and fourth flexor digitorum superficialis to the abductor pollicis longus and extensor pollicis brevis were performed. The skin defect was covered us-

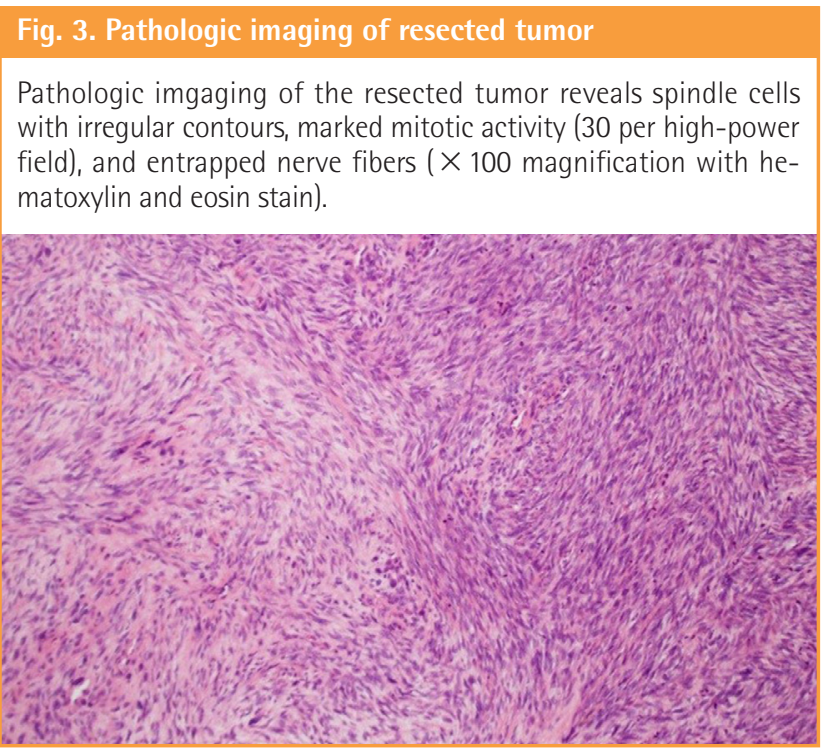

Fig. 4. Pinching motion at postoperative 22 months

Pinching with the thumb and little finger could be performed. Thumb abduction was preserved $\left(35^{\circ}\right)$.

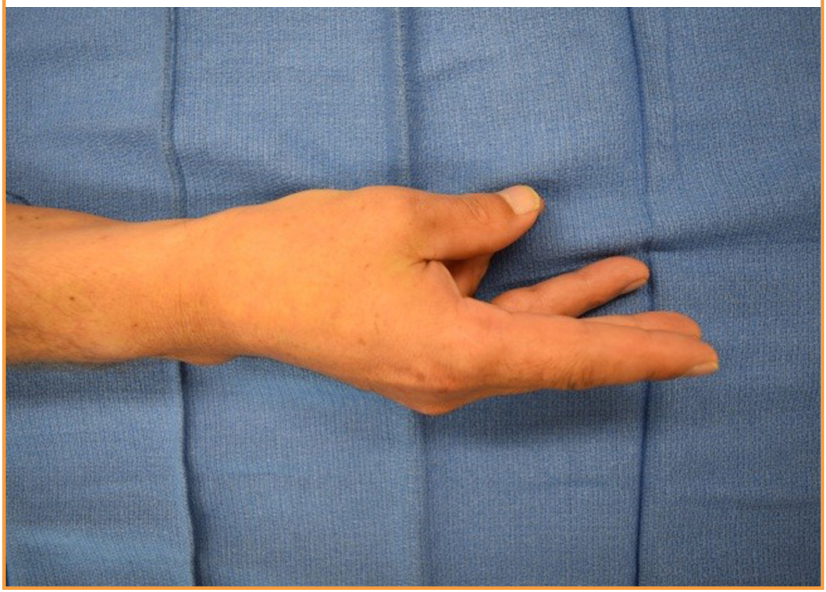

ing a $24 \times 8 \mathrm{~cm}$ left ALT perforator free flap (Fig. 2). The descending branch of the lateral circumflex femoral artery was anastomosed end-to-side with the radial artery. Two perforators from the vena comitans of the lateral circumflex femoral artery descending branch were anastomosed end-to-end with the cephalic vein and vena comitans. The sharp edges of the remnant radius were burred to prevent internal injury. The resected mass was confirmed to be a MPNST. Histologic findings revealed spindle cells with irregular contours, marked mitotic activity (30 per high-power field) and entrapped nerve fibers (Fig. 3). The patient was discharged after 18 days without any wound complications or hindered mobility. The arm was immobilized with a conventional long arm splint, and active flexion and extension exercises were started 4 weeks postoperatively. The patient returned to normal activity with full fist motion, pinch of the 


\section{Fig. 5. Fist motion at postoperative 22 months}

A full fist was made performed with full flexion of all five fingers.

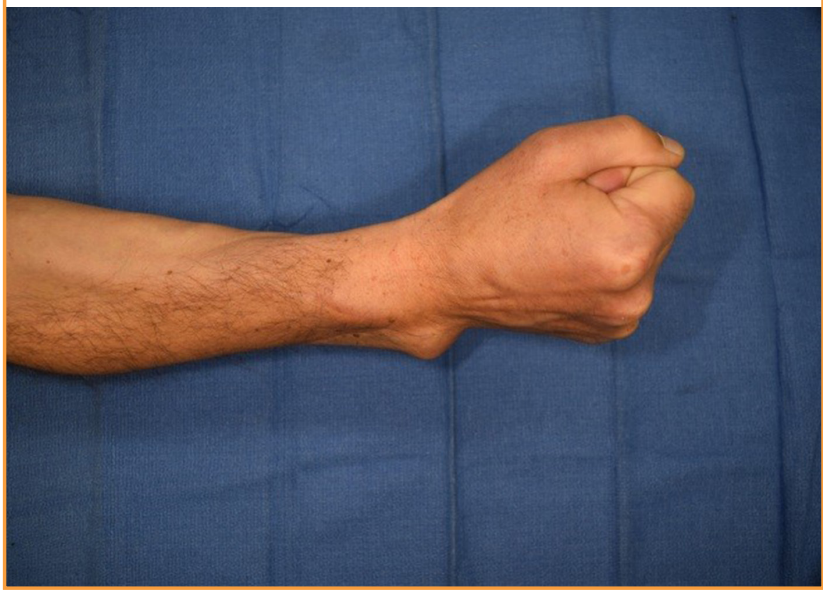

Fig. 6. Thumb hyperextension at postoperative 22 months

Thumb metacarpophalageal joint hyperextension was preserved $\left(10^{\circ}\right)$. All five fingers were extended.

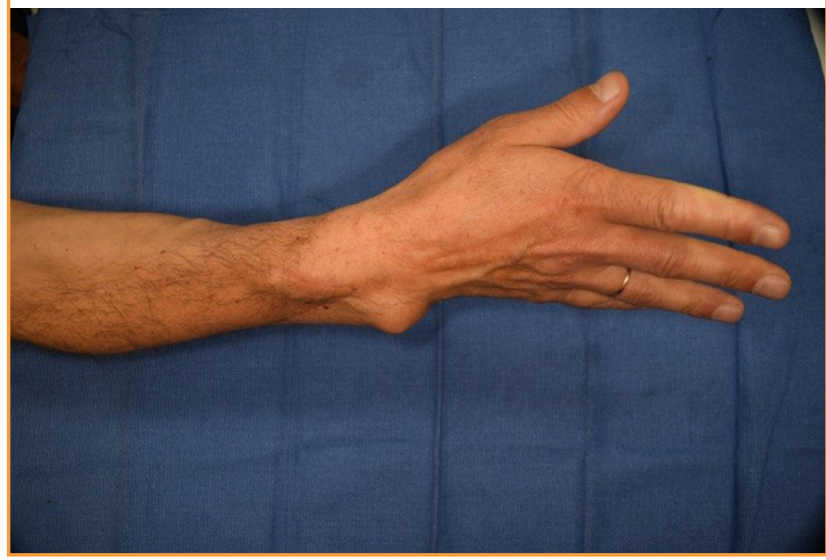

thumb and little finger, wrist extension of $65^{\circ}$, thumb metacarpophalangeal joint extension of $10^{\circ}$, and radial abduction of the thumb of $45^{\circ}$, in 22 months (Figs. 4-8). The American Society for Surgery of the Hand suggests that the normal range of motion is $70^{\circ}$ for wrist extension, $10^{\circ}$ for thumb metacarpophalangeal joint extension, and $60^{\circ}$ for radial abduction of the thumb.

\section{DISCUSSION}

MPNST is either associated with NF1 or occurs spontaneously. Fifty percent of MPNST patients have NF1, and 10\% have a history of radiation exposure [4]. The symptoms of MPNST are non-specific, other than nerve-related symptoms caused by tumor compression or infiltration. Patients present with mild pain, dysesthesia, or an asymptomatic mass [5]. Diagnosis can be delayed, which may be related to the poor prognosis. Distant
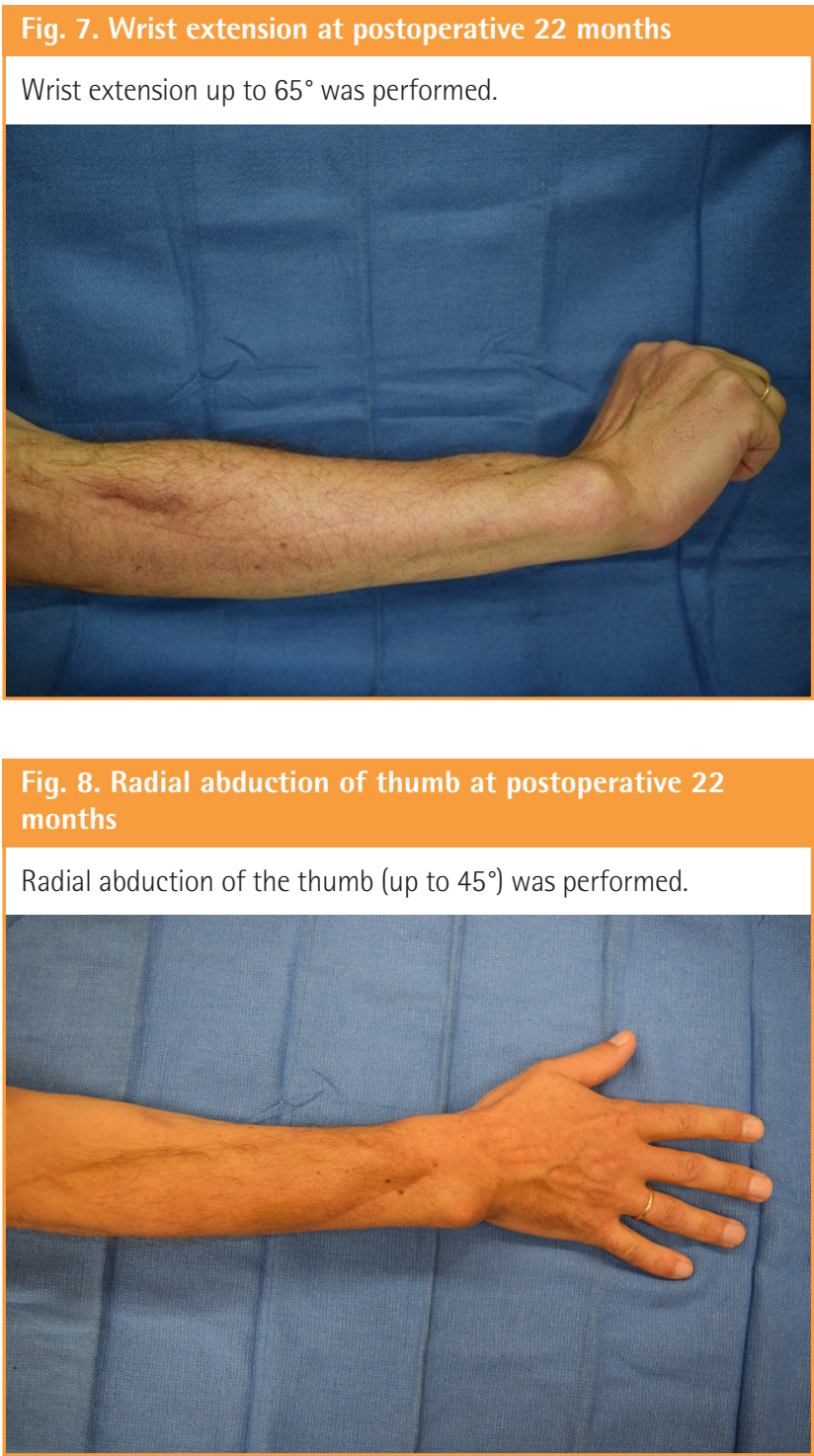

metastasis is an important factor to consider, as the 5-year survival of MPNST patients with distant metastasis has been reported to be as low as $18 \%$, while those without distant metastasis show a 5-year survival rate of 74\%. Fifty percent of MPNST patients suffer lung metastasis. Other common distant metastasis sites are the bones, mediastinum, brain, and liver [5].

MPNST is diagnosed based on pathologic reports, as there are no pathognomic molecular or immunohistochemistry studies [6]. Similar pathologic features are shown by several types of tumors, such as monophasic fibrotic synovial cell sarcoma, benign neurofibroma, malignant melanoma, dermatofibrosarcoma protuberans, ganglial neuroblastoma, synovial cell sarcoma, liposarcoma, leiomyosarcoma/non-Hodgkin lymphoma/malignant histiocytoma, leiomyosarcoma, and spindle cell myxoid pleomorphic sarcoma [4].

The poor prognosis, difficult diagnosis, and lack of a unified 
protocol due to the extremely low incidence of MPNST are troublesome. Extensive history-taking and imaging studies to assess the presence of distant metastasis are crucial $[4,5]$.

MPNST has a predilection for the extremities, whereas the head and trunk regions are much less frequently affected. While the prognosis of MPNST in the forearm has not been reported, sarcoma of the forearm tends to have a worse prognosis than similarly sized tumors in other anatomical locations, which leads to a tendency for wider resection [7]. Forearm reconstruction is challenging due to its unique anatomical structures. Hence, amputation of the affected limb has been the treatment of choice in the past [3].

Recent 5-year survival rates approaching $80 \%$ demand reconstruction techniques that are both aesthetically and functionally successful $[8,9]$. Limb-preserving operations showed no significant differences compared with amputation in terms of overall survival and disease-free interval [10]. Surgical outcomes need to be assessed both in terms of their oncological results and based on a consideration of the functional outcomes. An appreciation for the etiology and socioeconomic implications of upper extremity wounds is crucial for a successful outcome [11].

Soft-tissue coverage of defects affecting the hand, wrist, and elbow can be accomplished using skin grafts, local flaps, regional or distant pedicled flaps, and free flaps [12]. Resection of soft tissue sarcoma in the extremities frequently results in large complex soft tissue defects for which simple skin grafting or primary wound closure is impossible. Surgical margins should not be compromised to maintain function. ALT free flaps were first introduced by Song et al. [13] in 1984, and are advantageous for covering skin and soft-tissue defects due to their long vascular pedicle and abundant soft tissue volume. Two teams can work simultaneously in cases of a donor site on the upper extremity. One team can resect the tumor, while the other team harvests the flap [3]. Fasciocutaneous flaps provide adequate soft tissue coverage, a good contour, multiple possible orientations for flap inset, and a potential gliding surface for tendons [11].

The importance of upper extremity function contrasts with the scarcity of clinical reports of combined tendon transfers and free flap coverage. A critical issue is the timing of functional restoration. Simultaneous functional reconstruction and soft tissue coverage require fewer operations, with greater recovered range of motion and faster return to work [14]. Immediate functional restoration minimizes undesired changes of anatomic structures. Secondary functional reconstruction is accompanied by fibrotic changes and delayed wound healing, and it must be kept in mind that soft tissue sarcoma may require radiotherapy [15].

MPNST is a very rare type of tumor, especially in the extremities. There is no consensus in terms of operative criteria.
MPNST patients are burdened by both the malignancy as well as the resultant devastating impairment in their quality of life. With our simultaneous reconstruction method using an ALT free flap, the tumor itself can be optimally resected with no fear of extensive soft tissue damage that would prevent successful reconstruction and retention of function. To our knowledge, this is the first report describing reconstruction of the extensor compartment of the forearm with coverage using an ALT free flap after resection of an MPNST.

\section{NOTES}

\section{Conflict of interest}

No potential conflict of interest relevant to this article was reported.

\section{Ethical approval}

The study was approved by the Institutional Review Board of Hanyang University Hospital, Seoul (IRB No. 2018-04-026) and performed in accordance with the principles of the Declaration of Helsinki. Written informed consents were obtained.

\section{Patient consent}

The patient provided written informed consent for the publication and the use of his images.

\section{REFERENCES}

1. Wruhs M, Feldmann R, Breier F, et al. Malignant peripheral nerve sheath tumor in three patients of advanced age. J Dtsch Dermatol Ges 2016;14:72-4.

2. Singla P, Kachare SD, Fitzgerald TL, et al. Reconstruction using a pedicled upper arm fillet flap after excision of a malignant peripheral nerve sheath tumor: a case report. World J Clin Cases 2014;2:899-902.

3. Lee N, Roh S, Yang K, et al. Reconstruction of hand and forearm after sarcoma resection using anterolateral thigh free flap. J Plast Reconstr Aesthet Surg 2009;62:e584-6.

4. Farid M, Demicco EG, Garcia R, et al. Malignant peripheral nerve sheath tumors. Oncologist 2014;19:193-201.

5. Goertz O, Langer S, Uthoff D, et al. Diagnosis, treatment and survival of 65 patients with malignant peripheral nerve sheath tumors. Anticancer Res 2014;34:777-83.

6. Rodriguez FJ, Folpe AL, Giannini C, et al. Pathology of peripheral nerve sheath tumors: diagnostic overview and update on selected diagnostic problems. Acta Neuropathol 2012;123:295-319.

7. Lohman RF, Nabawi AS, Reece GP, et al. Soft tissue sarco- 
ma of the upper extremity: a 5-year experience at two institutions emphasizing the role of soft tissue flap reconstruction. Cancer 2002;94:2256-64.

8. Martinez SR, Robbins AS, Meyers FJ, et al. Racial and ethnic differences in treatment and survival among adults with primary extremity soft-tissue sarcoma. Cancer 2008;112: 1162-8.

9. Kim JY, Youssef A, Subramanian V, et al. Upper extremity reconstruction following resection of soft tissue sarcomas: a functional outcomes analysis. Ann Surg Oncol 2004;11: 921-7.

10. D’Orazi V, Panunzi A, Paoloni M, et al. Microsurgical approach for unusual and unexpected malignant fibrous histiocytoma of the forearm: a case report. Mol Clin Oncol 2015;3:1251-4.
11. Adkinson JM, Chung KC. Flap reconstruction of the elbow and forearm: a case-based approach. Hand Clin 2014;30: 153-63.

12. Jones NF, Jarrahy R, Kaufman MR. Pedicled and free radial forearm flaps for reconstruction of the elbow, wrist, and hand. Plast Reconstr Surg 2008;121:887-98.

13. Song YG, Chen GZ, Song YL. The free thigh flap: a new free flap concept based on the septocutaneous artery. Br J Plast Surg 1984;37:149-59.

14. Sundine M, Scheker LR. A comparison of immediate and staged reconstruction of the dorsum of the hand. J Hand Surg Br 1996;21:216-21.

15. Delanian S, Lefaix JL. The radiation-induced fibroatrophic process: therapeutic perspective via the antioxidant pathway. Radiother Oncol 2004;73:119-31. 